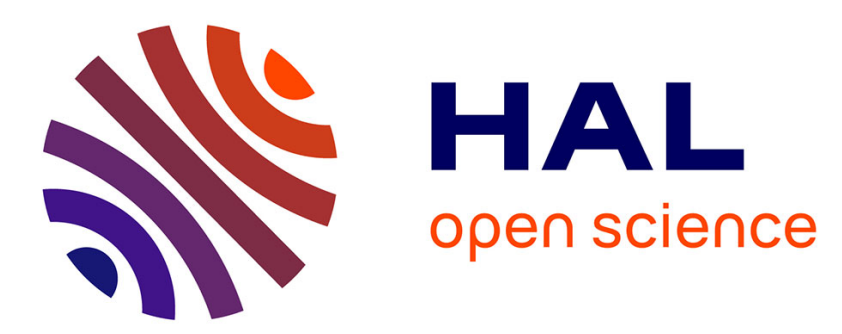

\title{
Français, dialectes galloromans et di(a)glossie : présentation
}

\author{
Mathieu Avanzi, André Thibault
}

\section{To cite this version:}

Mathieu Avanzi, André Thibault. Français, dialectes galloromans et di(a)glossie : présentation. Langages, 2019, $\mathrm{N}^{\circ} 215$ (3), pp.9. 10.3917/lang.215.0009 . hal-02531918

\section{HAL Id: hal-02531918 https://hal.sorbonne-universite.fr/hal-02531918}

Submitted on 12 Apr 2020

HAL is a multi-disciplinary open access archive for the deposit and dissemination of scientific research documents, whether they are published or not. The documents may come from teaching and research institutions in France or abroad, or from public or private research centers.
L'archive ouverte pluridisciplinaire HAL, est destinée au dépôt et à la diffusion de documents scientifiques de niveau recherche, publiés ou non, émanant des établissements d'enseignement et de recherche français ou étrangers, des laboratoires publics ou privés. 


\title{
Français, dialectes galloromans et di(a)glossie : présentation
}

\author{
Mathieu Avanzi \\ Sorbonne Université \\ André Thibault \\ Sorbonne Université
}

Le français dans la francophonie d'Europe est caractérisé par une longue coexistence avec les parlers galloromans, "dialectes primaires" dans la terminologie de E. Coseriu (1980), alors que les différentes variétés régionales résultant de la diversification du français lui-même sur l'axe diatopique, au gré de son expansion géographique, sont considérées dans ce même modèle comme des "dialectes secondaires " parfois aussi appelés « régiolectes » (v. encore Glessgen \& Thibault 2005 sur la régionalité linguistique dans la Romania et en français). Une certaine tradition, naguère fortement représentée au sein des dialectologues du galloroman, cherchait à expliquer toute la variation diatopique du français et de ses "régiolectes » par l'influence des patois isotopes, niant ainsi toute dynamique interne au français dans son expansion tout à la fois horizontale (régionale) et verticale (sociale). En outre, une telle vision des choses empêchait par définition d'évaluer l'effet inverse, c'est-à-dire l'influence du français sur les patois (sur celle-ci, v. entre autres Chambon 2009 et Kristol 2009), longtemps confinée dans l'angle mort des chercheurs. Or, cette conception a été remise en question par de nombreux auteurs (v. déjà Bloch 1921 pour un prédécesseur, et Chambon \& Greub 2009 pour une vue d'ensemble) qui ont démontré d'une part que le français jouit d'un dynamisme propre et d'autre part qu'il peut fortement influencer les patois avec lesquels il a coexisté pendant des siècles, allant d'ailleurs en dernière analyse jusqu'à les faire disparaître ${ }^{1}$.

Il ne s'agit d'ailleurs pas de tomber dans l'excès contraire et de nier toute influence «substratique» ou «adstratique» dans la configuration des régiolectes, mais bien plutôt d'identifier avec précision la nature et l'envergure de ces influences, ainsi que leur fonctionnement et la direction

\footnotetext{
${ }^{1}$ On notera d'ailleurs que les études sur l'étiolement et la mort des langues («language attrition » et « language death », v. Dorian dir. 1989 et Valdman 1997), qui devraient normalement trouver un terrain d'étude idoine en Galloromania, restent très peu représentées en France - et ce pour des raisons probablement liées à la psychologie des chercheurs : ni l'étude de la francisation des parlers, ni celle de leur lente disparition, ne semblent être des sujets susceptibles de soulever l'enthousiasme des galloromanistes.
} 
dans laquelle elles s'exercent. Dans le domaine du lexique (toujours encadré par le garde-fou constitué par la phonétique historique des parlers), J.-P. Chauveau (2005) offre une bonne démonstration du travail d'orfèvre qu'il faut consentir pour dresser un portrait fidèle et précis de ce qu'il appelle les " différents étages d'une maison communiquant par des escaliers qui font partie constitutive de la construction» (Chauveau, 2005: 44), image qu'il oppose à celle, un peu trop rigide, de strates géologiques plus ou moins fortement imperméables.

M. Francard (2005) aborde la question d'un point de vue sociolinguistique : il est le premier en contexte galloroman à avoir attiré l'attention sur les propositions de P. Auer $(1998,2005)$ qui tente de modéliser les différents types de contacts entre les dialectes primaires et leur langue-toit (< all. Dachsprache, Kloss 1987) à l'échelle européenne. Cet auteur identifie quatre cas de figure possibles (à la suite du type 'Zéro' correspondant à l'opposition médiévale entre le latin écrit et les langues vulgaires n'ayant encore subi aucune amorce de standardisation) : le type A, celui d'une diglossie classique (Ferguson 1959), où des dialectes confinés à l'oralité sont chapeautés par un standard 'endoglossique' (c'est-à-dire étroitement apparenté) principalement conçu pour les besoins de l'écrit; le type $B$, une forme atténuée de diglossie, caractérisée par un début d'expansion du standard écrit au discours oral spontané, en particulier dans des interactions marquées par un certain degré de formalité et limitées à des domaines bien définis; le type $\mathrm{C}$, que $\mathrm{P}$. Auer appelle «diaglossie» à la suite de G. Bellmann (1997), et dont le trait définitoire est qu'il présente une situation de continuum entre le standard d'une part et les dialectes les plus éloignés de celui-ci d'autre part, à l'intérieur duquel les locuteurs évoluent aisément (« speakers can change their way of speaking without a clear and abrupt point of transition between dialect and standard » Auer, $2005: 23)$; enfin, le type $\mathrm{D}$, correspondant à la disparition totale du dialecte au profit du standard - et ce, à partir d'une situation diglossique ou diaglossique. Dans une optique diachronique, les types identifiés par P. Auer se succèdent dans le temps (A, B, C et D) de façon graduelle, mais des étapes peuvent être sautées ou jamais atteintes selon les différentes configurations nationales.

L'article de P. Auer (2005) est très bien documenté en ce qui concerne les langues germaniques et les langues slaves; au sein des langues romanes, l'italien, l'espagnol et le portugais sont souvent mentionnés. En revanche, le français y fait figure de parent pauvre : un seul titre dans une bibliographie qui en compte plus de 80 . Il convient donc de combler cette lacune et d'examiner dans quelle mesure la modélisation en question peut s'appliquer à l'espace et à l'histoire du galloroman. P. Auer lui-même classe le français dans la catégorie $\mathrm{D} / 1$, c'est-à-dire " from diaglossia to dialect loss ", mais à vrai dire la question de savoir si l'on est arrivé à la perte des dialectes à 
partir d'une situation de diglossie, de diglossie atténuée ou de diaglossie pose problème - d'une part, parce que la frontière entre ces cas de figure est graduelle, et d'autre part parce que l'espace galloroman se scinde en trois grands ensembles (oïl, francoprovençal et oc) que l'on ne peut pas mettre sur le même pied.

C'est en domaine d'oïl que la distance typologique entre le français et ses plus proches cousins est la moins importante, ce qui peut théoriquement faciliter l'émergence d'un continuum diaglossique. M. Francard (2005: 58) postule pour la Wallonie une situation de «diglossie atténuée» qui aurait prévalu jusqu'au début du $\mathrm{XX}^{\mathrm{e}}$ siècle, «alors que, dans d'autres régions du domaine d'oïl, la diaglossie était déjà sur le déclin». Il précise : «la persistance d'une diglossie en Wallonie, processus auquel l'imposition de l'instruction primaire a mis fin assez brutalement et tardivement (à partir de 1920), a réduit la durée de l'étape de diaglossie, et son extension dans la population ». Cela dit, rien ne permet d'affirmer que le reste du domaine d'oïl aurait connu une longue période de «diaglossie » caractérisée par un continuum entre deux pôles. D'autres modèles sont possibles, comme par exemple celui de l'alternance codique (selon le sujet abordé et/ou l'interlocuteur, comportement encore observable in vivo); J.-P. Chambon / Y. Greub (2009: 2555) évoquent «la formation transitoire de variétés d'apprenants » et « la formation éventuelle d'interlangues », ce qui revient à dire que pour eux l'étape de «diaglossie», bien qu'envisageable, ne saurait être que très brève ou encore anecdotique. Nous manquons cruellement de données textuelles de nature épilinguistique nous permettant de mieux connaître le comportement langagier des locuteurs oïliques d'autrefois, et il est difficile de le reconstruire à partir des sources disponibles documentant les patois d'une part et le français régional d'autre part, ces sources devant elles-mêmes être abordées avec méfiance et de façon critique (cf. contribution d'E. Baiwir dans ce recueil). Quant au francoprovençal et à l'occitan, la distance typologique qui les sépare du français rend tout de suite plus improbable l'instauration d'une longue période de " diaglossie » où l'on aurait massivement parlé en société une sorte de code intermédiaire - notons en tout cas que l'existence de tels codes hybrides n'est pas du tout documentée, et l'on se gardera bien de prétendre que le français régional du sud (peu importe l'intensité de l'accent) peut être considéré comme typologiquement à mi-chemin entre le français et l'occitan.

Autant dire que devant la rareté des données disponibles, une bonne partie du travail de description des rapports historiques entre le français et les dialectes galloromans, autant sur le plan interne (systémique) qu'externe $(\text { social })^{2}$, reste à faire. Le présent recueil a pour ambition d'apporter une

\footnotetext{
${ }^{2}$ On consultera avec profit G. Kremnitz et al. (2013) pour une introduction d'ensemble à l'histoire de l'architecture sociale des langues de France. Sur cet ouvrage, v. G. Siouffi (2015) et A. Thibault (2016).
} 
petite pierre à cet édifice. Il s'ouvre sur une contribution de Y. Greub qui s'attaque à la délicate question de la frontière entre français et francoprovençal à l'époque médiévale, lorsqu'il s'agit de catégoriser des productions écrites qui sont par définition un artefact résultant de nombreux compromis. Non seulement est-il difficile d'identifier les variétés, mais l'attribution de variantes à l'une ou l'autre des variétés postulées (et reconstruites) pose des problèmes encore plus aigus. La contribution suivante, signée par E. Baiwir, propose une approche critique des sources dialectales, en l'occurrence les atlas linguistiques. Il risque d'être ardu (voire trompeur) de se baser sur ces sources pour appréhender la nature plus ou moins di(a)glossique du comportement langagier des communautés oïliques, dans la mesure où il semble avéré que les atlantographes ont volontairement construit un état "figé" des dialectes, en accord avec la conception « puriste » qu'ils s'en faisaient. L'article de M. Bergeron se penche sur trois variables repérées dans des textes écrits par des peu-lettrés au $18^{\mathrm{e}}$ siècle en Haute-Normandie, et pouvant être considérées comme des «formes transitoires " attestant d'un processus d'appropriation du français par les populations dialectophones de l'époque - des traces, en somme, de leur interlecte d'apprenants. Les deux contributions suivantes sont consacrées à des phénomènes grammaticaux situés à l'interface entre français et patois, que l'influence se soit exercée dans un sens ou dans l'autre. M. Duval montre l'impact de la morphologie du verbe français sur celle des formes relevées dans les patois lorrains, plus précisément dans les environs de Metz, centre historique de diffusion du français mais aussi du dialecte local dans la région. Y. Ch. Morin propose une étude de la gémination du pronom clitique le dans le français poitevin du tournant $\mathrm{du} \mathrm{XVI}^{\mathrm{e}}$ siècle, qu'un examen minutieux des données dialectales permet d'expliquer comme le résultat d'un effet adstratique. On passe à une problématique plus lexicale avec M. Avanzi et A. Thibault, qui invitent à jeter un regard neuf sur l'histoire des adjectifs cardinaux (respectivement décimaux et vigésimaux) servant à exprimer les nombres 70, 80 et 90 en français ainsi que dans les parlers galloromans; on découvrira que l'influence ne s'exerce pas nécessairement dans le sens où on l'attendrait. Dans la dernière contribution, W. Remysen se consacre à la façon dont la dichotomie relativement moderne "dialectes primaires» vs. «dialectes secondaires》 a été interprétée par la tradition historiographique canadienne-française, dans son appréhension de la genèse du français québécois et de ses origines. 


\section{Références}

AUER P. (éd.) (1998), Folia Linguistica 32, 1-2: Dialect levelling and the standard varieties in Europe.

AuER P. (1998), "Dialect levelling and the standard varieties in Europe", in id. (éd.), 1-9.

AUER P. (2005), "Europe's sociolinguistic unity, or: a typology of European dialect/standard constellations" in N. Delbecque, J. van der Auwera \& D. Geeraerts (éds), Perspectives on variation, Berlin / New York : de Gruyter, 7-42.

Bellmann G. (1997), "Between Base Dialect and Standard Language", Folia Linguistica 32, $1-2,23-34$.

BLOCH O. (1921), La pénétration du français dans les parlers des Vosges méridionales, Paris : Champion.

Chambon J.-P. (1997), "Les emprunts du français moderne aux dialectes ou patois: une illusion d'optique en lexicologie française ou historique ? », Lalies, 33-53.

ChAmBon J.-P. (2009), «Prolégomènes à l'étude historique des emprunts de l'occitan au français ", in A. Thibault (éd.), Gallicismes et théorie de l'emprunt linguistique, Paris : L'Harmattan, 45-69.

Chambon J.-P. \& GReub Y. (2009). « Histoire des variétés régionales dans la Romania: français », in G. Ernst, M.-D. Gleßgen, Ch. Schmitt \& W. Schweickard (éds), Handbücher zur Sprach- und Kommunikationswissenschaft, Berlin/New York: de Gruyter, 2552-2565.

Chauveau J.-P. (2005), « Régionalismes et dialectalismes : quelques exemples manceaux », in M.-D. Glessgen \& A. Thibault (éds), La lexicographie différentielle du français et le Dictionnaire des régionalismes de France, Actes du Colloque en l'honneur de Pierre Rézeau, Strasbourg : Presses Universitaires de Strasbourg, 31-44.

Coseriu E. (1980), «"Historische Sprache” und "Dialekt” », in J. Göschel, P. Ivić \& K. Kehr (éds), Dialekt und Dialektologie, Wiesbaden : Steiner, 106-122.

Dorian N. C. (dir.) (1989), Investigating Obsolescence: Studies in Language Contraction and Death, Cambridge : Cambridge University Press.

FERguson Ch. A. (1959), "Diglossia", Word 15, 325-340.

FRANCARD M. (2005), «La frontière entre les langues régionales romanes et le français en Wallonie », in M.-D. Glessgen \& A. Thibault (éds), La lexicographie différentielle $d u$ français et le Dictionnaire des régionalismes de France, Actes du Colloque en l'honneur de Pierre Rézeau, Strasbourg : Presses Universitaires de Strasbourg, 45-61.

Glessgen M.-D. \& Thibault A. (2005), «La 'régionalité linguistique' dans la Romania et en français ", in idd. (éds), La lexicographie différentielle du français et le Dictionnaire des régionalismes de France, Actes du Colloque en l'honneur de Pierre Rézeau, Strasbourg : Presses Universitaires de Strasbourg, III-XVII.

KLoss H. (1987), « Abstandsprache und Ausbausprache », in U. Ammon et al. (éd.), Sociolinguistics / Soziolinguistik, vol. 1, Berlin/New York : de Gruyter, 302-308.

Kremnitz G. (dir.), avec le concours de F. Broudic et al. (2013), Histoire sociale des langues de France, Rennes: Presses Universitaires de Rennes.

KRIStol A. M. (2009), «Contact des langues et emprunt. L'influence du français sur les parlers galloromans de la Suisse romande », in A. Thibault (éd.), Gallicismes et théorie de l'emprunt linguistique, Paris : L'Harmattan, 71-91.

Siouffi G. (2015), compte rendu de Kremnitz et al. 2013, Revue de linguistique romane 79, 249-253. 
Thibault A. (2016), compte rendu de Kremnitz et al. 2013, Bulletin de la Société de linguistique de Paris 111/2, 87-94.

VAldman A. (1997), «Étiolement linguistique », in M.-L. Moreau (éd.), Sociolinguistique. Les concepts de base, Sprimont : Mardaga, 144-151. 